\title{
Anticuerpos contra Leptospira spp en caprinos lecheros en Guanajuato, México
}

\author{
Antibodies against Leptospira spp in dairy goats in Guanajuato, Mexico \\ Miguel Ángel Luna Álvarez ${ }^{1,4}$, Guadalupe Socci Escatell ${ }^{1}$, José Javier Morales \\ Arzate $^{2}$, José Manuel Oliveros Ibarra ${ }^{2}$, Eva María Luna Rivera ${ }^{3}$
}

\section{Resumen}

El estudio tuvo como objetivo determinar el perfil serológico de anticuerpos contra leptospirosis en dos unidades caprinas de producción lechera en Apaseo el Alto, municipio de Guanajuato, México en 2014. Se trabajó con animales de las razas Saanen, Alpina, Nubia y Toggenburg categorizados por edad para el muestreo aleatorio. Para la detección de anticuerpos contra Leptospira se utilizó la prueba de aglutinación microscópica (MAT) con antígeno vivo propuesta por la Organización Mundial de Sanidad Animal (OIE) y la Organización Mundial de la Salud(OMS). Se utilizaron 12 serovariedades de Leptospira spp, y se consideraron como positivos los sueron con títulos iguales o mayores a 1:100. El 68.3\% (41/60) de las muestras de la Unidad «A» resultaron positivas, con títulos entre 1:100 y 1:1600, en tanto que el 72.4\% (97/134) de las muestras de la Unidad «B» resultaron positivas, con títulos entre 1:100 y 1:800. La frecuencia de animales positivos por edad varió entre $20 \%$ (en el caso de cabritos) y $100 \%$ (en animales adultos). Se concluye que los anticuerpos anti-Leptospira fueron altos y que se generaron por un proceso infeccioso, dado que los animales nunca fueron vacunados contra la enfermedad.

Palabras clave: leptospirosis; abortos; serología; caprinos; zoonosis

\footnotetext{
${ }^{1}$ Laboratorio de Leptospirosis, CENID-Microbiología Animal, Instituto Nacional de Investigaciones Forestales y Agropecuarias (INIFAP), Palo alto CDMX, México

${ }^{2}$ Secretaría de Agricultura, Ganadería, Pesca y Alimentación (SAGARPA), Guanajuato, México

${ }^{3}$ Laboratorio de Biología Molecular, Hospital Central Sur Pemex-Picacho, CDMX, México

${ }^{4}$ E-mail: luamvet@gmail.com

Recibido: 1 de febrero de 2018

Aceptado para publicación: 2 de abril de 2018
} 
The study aimed to determine the serological profile of antibodies against leptospirosis in two dairy goat units in Apaseo el Alto, Guanajuato, Mexico in 2014. Saanen, Alpine, Nubian and Toggenburg goats were classified according to age for random sampling. The microscopic agglutination test (MAT) with live antigen proposed by the World Organisation for Animal Health (OIE) and the World Health Organization (WHO) were used for detecting anti-Leptospira antibodies. Twelve serovars of Leptospira spp were used and animals with equal or higher titres of 1:100 were considered positive. Results showed that $68.3 \%$ (41/60) of samples of Unit «A» were positive, with titres from 1:100 to 1: 1600 , while $72.4 \%$ (97/134) of samples of Unit «B» were positive, with titres between 1:100 to 1:800. Frequencies of positive animals according to age ranged from $20 \%$ in kids and $100 \%$ in adults. It is concluded that the anti-Leptospira antibodies were high and were generated by an infectious process since the animals were never vaccinated against the disease.

Key words: leptospirosis; abortions; serology; goats; zoonosis

\section{INTRODUCCIÓN}

La leptospirosis es una enfermedad de origen animal que puede afectar accidentalmente al hombre. Es causada por bacterias del género Leptospira, especie $L$. interrogans. Se caracteriza por provocar trastornos de tipo reproductivo en el ganado, tales como reabsorciones embrionarias, abortos, retenciones placentarias, mortinatos y muerte perinatal. La bacteria penetra al organismo del hospedero a través de mucosas y piel lacerada o reblandecida. Asimismo, puede ser eliminada a través de la orina y secreciones pos-aborto de animales infectados (Acha y Szyfres, 2001; Adler y De la Peña, 2010).

En el ser humano, la leptospirosis o enfermedad de Weil es capaz de ocasionar trastornos febriles, hepático-renales, ictericia, neumonías, hemorragias pulmonares, hemoptisis, trastornos circulatorios, insuficiencia cardiaca y muerte (Zavala et al., 2008; Seijo et al., 2011). En México, el Sector Salud, por medio de la Secretaria de Salubridad y Asistencia, la consideró una enfermedad de im- portancia por estar presente a nivel mundial en poblaciones marginadas por pobreza y en zonas carentes de servicios públicos, así como por presentarse en desastres naturales tales como inundaciones y terremotos (Acha y Szyfres, 2001). Es así, que se establecieron una serie de estrategias de control en la norma oficial mexicana NOM-029-SSA2-1999, para la vigilancia epidemiológica, prevención y control de la leptospirosis humana y la incluyó como zoonosis en la NOM-017-SSA2012, para la vigilancia epidemiológica. Así como en el proyecto de norma oficial mexicana PROY-NOM-029-SSA2-2014, para la prevención y control de la leptospirosis en el humano.

Con respecto a la salud animal, el impacto de la leptospirosis como enfermedad reproductiva se ha visto reflejada principalmente en el ganado bovino, donde la Secretaría de Agricultura, Ganadería, Desarrollo Rural, Pesca y Alimentación (SAGARPA) desarrolló estrategias para su control, descritas en la norma oficial mexicana NOM-038ZOO-1995 requisitos mínimos para la elaboración de bacterinas empleadas en la prevención y control de la leptospirosis bovina 
(NOM, 1996). Sin embargo, se desconoce la importancia de esta enfermedad en otras especies de interés productivo como en pequeñs rumiantes. En caprinos no solo se carece de normas o estrategias preventivas para su control, sino que además no existe un producto biológico en el país para prevenir la enfermedad. Asimismo, se desconoce el papel de la cabra como reservorio y transmisor de la infección a otras especies, incluyendo al hombre. La literatura especializada únicamente menciona la similitud que existe con la leptospirosis bovina y ovina, y reporta a la cabra como una de las especies más 'resistentes' a la infección (Manual Merck, 1981; Faine, 1982; Hagan y Bruner, 1983). No obstante, algunos autores difieren en este concepto y consideran a la leptospirosis en pequeños rumiantes como una enfermedad relevante que provoca un impacto negativo en la productividad de los rebaños (Lilenbaum et al., 2007).

El objetivo del presente estudio fue determinar la presencia de anticuerpos contra Leptospira spp en suero de cabras lecheras, utilizando la técnica diagnóstica de referencia internacional de aglutinación microscópica (MAT).

\section{Materiales y Métodos}

Se realizó un muestreo transversal por oportunidad en animales de dos unidades de producción caprina especializadas en la producción de leche, localizadas en Apaseo el Alto, municipio de Guanajuato, México en 2014. Las unidades contaban con certificado de «rebaño libre de Brucella» y realizaban control de Clamydia, pero continuaban presentando trastornos reproductivos persistentes. En estas unidades no se vacuó contra leptospirosis. Las cabras fueron de las razas Saanen, Alpina, Nubia y Toggenburg.
Los animales fueron estratificados por edad (2, 3, 4, 5, 6 y 9 meses; 1, 2, 3, 5 años y sementales) para el muestreo aleatorio.

El tamaño de muestra se obtuvo considerando una prevalencia de la enfermedad del $60 \%$ según lo reportado para ganado ovino en la región (Luna et al., 2014). En la unidad «A» se tomaron 60 muestras de un total de 240 animales $(25 \%)$ y en la unidad «B» se tomaron 134 muestras de 400 animales $(33.5 \%)$. Las muestras se tomaron de la vena yugular en tubos tipo vacuntainer y fueron centrifugadas a $3000 \mathrm{rpm}$. Los sueros se decantaron en crioviales y fueron mantenidos a $-20{ }^{\circ} \mathrm{C}$ hasta su uso en el laboratorio.

Para la detección de anticuerpos contra Leptospira se utilizó la prueba diagnóstica de micro-aglutinación (MAT), propuesta por la Organización Mundial de Sanidad Animal (OIE, 2008) y la Organización Mundial de la Salud (OMS, 2013), utilizando 12 serovariedades de Leptospira spp (Cuadro 1). De estas, nueve serovariedades eran de referencia internacional, provenientes del Collaborating Centre for Reference and Research on Leptospirosis (Queensland, Australia) y tres serovariedades aisladas en la región norte y centro de México, tipificadas en el Departamento de Agricultura de Estados Unidos de Norteamérica (Dra Carole A. Bolin, USDA, Ames, Iowa, 1993).

Los sueros fueron trabajados en diluciones de 1:50 en adelante hasta dilución final, en placas de 96 pozos y las reacciones fueron observadas al microscopio de campo oscuro (Carl Zeiss Axiostar Plus, Alemania) a 160X. Todas las pruebas se realizaron en el laboratorio de leptospirosis del Centro Nacional de Investigación Disciplinaria en Microbiología Animal (CENID-Microbiología Animal) del Instituto Nacional de Investigaciones Forestales y Agrícolas y Pecuarias (INIFAP) en Ciudad de México. 
Cuadro 1. Serovariedades de Leptospira de referencia internacional ${ }^{1} \mathrm{y}$ de aislados nacionales ${ }^{2}$ utilizados como antígenos para el diagnóstico en la prueba de MAT

\begin{tabular}{|c|c|c|c|}
\hline Especie & Serogrupo & Serovariedad & Cepa \\
\hline L. interrogans ${ }^{1}$ & Icterohaemorrhagiae & Icterohaemorrhagiae & RGA \\
\hline L. interrogans ${ }^{1}$ & Pyrogenes & Pyrogenes & Salinem \\
\hline L. kirshneri ${ }^{1}$ & Grippotyphosa & Grippotyphosa & Moska V \\
\hline L. interrogans ${ }^{1}$ & Canicola & Canicola & Hond Utrecht IV \\
\hline L. interrogans ${ }^{1}$ & Pomona & Pomona & Pomona \\
\hline L. interrogans ${ }^{1}$ & Sejroe & Wolffi & 3705 \\
\hline L. interrogans ${ }^{1}$ & Sejroe & Hardjo & Hardjoprajitno \\
\hline L. borgpetersenii ${ }^{1}$ & Tarassovi & Tarassovi & Perepelicin \\
\hline L. interrogans ${ }^{1}$ & Australis & Bratislava & Jez-Bratislava \\
\hline L. interrogans ${ }^{2}$ & Sejroe & Hardjo & H-89 \\
\hline L. interrogans ${ }^{2}$ & Icterohaemorrhagie & Icterohaemorrhagiae & Palo Alto \\
\hline L. interrogans ${ }^{2}$ & Canicola & Portland-vere & Sinaloa ACR \\
\hline
\end{tabular}

${ }^{1} \mathrm{http} / / /$ www.pasteur.fr/recherche/Leptospira

Cuadro 2. Distribución porcentual de los títulos de anticuerpos contra leptospirosis de casos positivos $(\geq 1: 100)$ en dos unidades de producción caprina, en Apaseo el Alto, municipio de Guanajuato, México (2014)

\begin{tabular}{clcc}
\hline Título de anticuerpos & $\begin{array}{c}\text { Interpretación del título de } \\
\text { anticuerpos }\end{array}$ & $\begin{array}{c}\text { Unidad “A" } \\
(\%)\end{array}$ & $\begin{array}{c}\text { Unidad "B" } \\
(\%)\end{array}$ \\
\hline $1: 100$ & Positivo/bajo & 51.3 & 65.6 \\
$1: 200$ & Positivo /bajo & 28.2 & 16.4 \\
$1: 400$ & Positivo alto/moderado & 17.9 & 16.9 \\
$1: 800$ & Positivo alto /moderado & 1.3 & 1.1 \\
1:1600 & Positivo alto & 1.3 & 0.0 \\
\hline
\end{tabular}

${ }^{1}$ Zamora y Riedemann (1986)

Los criterios de evaluación e interpretación de los títulos de anticuerpos fueron divididos en: a) Evento negativo o sueros sin reacción; b) Evento sospechoso: sueros con títulos 1:50; c) Evento positivo: sueros con títulos $\geq 1: 100$. Asimismo, los títulos de 1:100 a 1:200 fueron considerados positivos bajos e indicativos de posible infección, de 1:400 a 1:800 como positivos altos/moderados e indicativos de infección y de 1:1600 en adelante como positivos altos e indicativos de infección y posible estado de portador (Zamora y Riedemann, 1986). 


\section{Resultados y Discusión}

El 68.3\% (41/60) de las muestras de cabras de la unidad «A» resultaron positivas a anticuerpos contra leptospirosis. Por otra parte, el 72.4\% (97/134) de las muestras de cabras de la unidad «B» fueron positivas (Figura 1). Los títulos de anticuerpos de los animales de la unidad «A» oscilaron entre 1:100 y $1: 1600$, y los de la unidad «B $»$ entre $1: 100$ y 1:800 (Cuadro 2).

Las frecuencias de animales positivos por grupo etario en las dos unidades en estudio se presentan en la Figura 2. Las frecuencias variaron entre 20 a $100 \%$. La menor frecuencia se presentó en hembras de dos meses de edad y se fue incrementando con la edad de las cabras, alcanzando el $100 \%$ en animales de 9 a 12 meses. Asimismo, se observó una disminución en la frecuencia en animales de 2-3 años. Los sementales presentaron una seropositividad que varió de $54.5 \%$ (Unidad «B») a 90\% (Unidad «A »).

Las muestras de animales de la Unidad «A» reaccionaron con títulos positivos contra cinco serovariedades, siendo las más frecuentes Icterohaemorrhagiae cepa Palo Alto (aislado), Canicola Portland-vere (aislado), Hardjo cepa H-89 (aislado), Grippotyphosa (Figura 3). Los animales de la unidad «B» reaccionaron contra seis serovariedades, siendo las más frecuentes Bratislava, Icterohaemorrhagiae cepa Palo Alto (aislado), Canicola Hond Utrecht IV y Canicola serovariedad Portland-vere (aislado) (Figura 3).

La respuesta serológica positiva y las serovariedades involucradas permite inferir que la infección está afectando animales de ambos rebaños en forma similar; lo cual podría deberse a la cercanía de instalaciones (menor a $1 \mathrm{~km}$ ) así como al préstamo, manejo y compra venta de animales que se realiza entre los propietarios de ambas unidades de producción.
Con respecto a la frecuencia de anticuerpos en los primeros 3-4 meses de vida se debe considerar la protección pasiva transmitida por las madres, la cual ocurre hasta los 4 meses en bovinos (Rebhum, 1999; Gómez, 2005), debiendo disminuir con el avance de la edad. Sin embargo, en este estudio se observó lo contrario, pues la frecuencia y los títulos de anticuerpos se incrementan de los 5-6 meses en adelante, en respuesta a una posible infección en el rebaño. A partir del año de edad, los títulos se mantienen oscilantes, debido probablemente a la circulación de la bacteria dentro del rebaño y a la respuesta inmune del organismo en un esfuerzo por controlar la infección. Los títulos en animales de 2-3 años son interrogantes al encontrarse disminuidos probablemente por ser hembras gestantes y tener disminución inmunológica para la implantación correcta del producto $\mathrm{y}$ «secuestro de anticuerpos» por la glándula mamaria al prepararse para la lactación (Mallard et al., 1998).

Con respecto a la alta frecuencia de serovariedades como Icterohaemorrahagiae, Bratislava y Canicola y cuyas especies portadoras en la naturaleza son ratas y perros, se reportó la observación de ratas en bodegas y dentro de los corrales. La presencia de perros dentro de las granjas también fue común, al ser utilizados como mascotas y guardianes de las instalaciones por los propietarios de las granjas en estudio.

Las reacciones cruzadas, coaglutinación o reacciones paradójicas contra 3, 4 o más serovariedades tienden a ser frecuentes en sueros positivos altos en la técnica de MAT; es así que mientras más altos son los títulos positivos, más reacciones de coaglutinación tienden a observarse debido a los antígenos de superficie que comparten las bacterias del género Leptospira (Levett, 2001).

Se concluye que fue posible detectar la presencia de anticuerpos anti-leptospira por la prueba de MAT en los rebaños muestreados. Estos fueron generados por un 


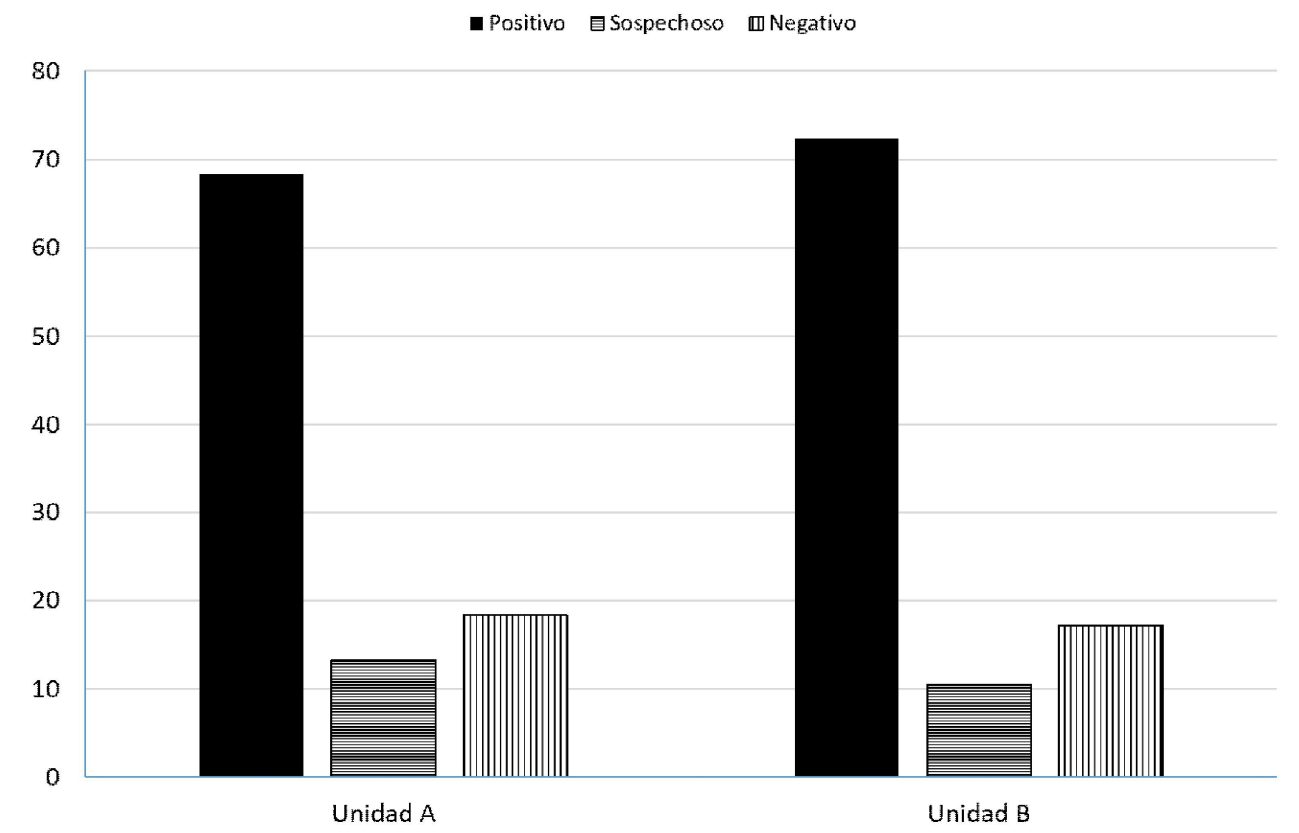

Figura 1. Frecuencia (\%) de leptospirosis caprina en dos unidades de producción en Apaseo el Alto, municipio de Guanajuato, México (2014)
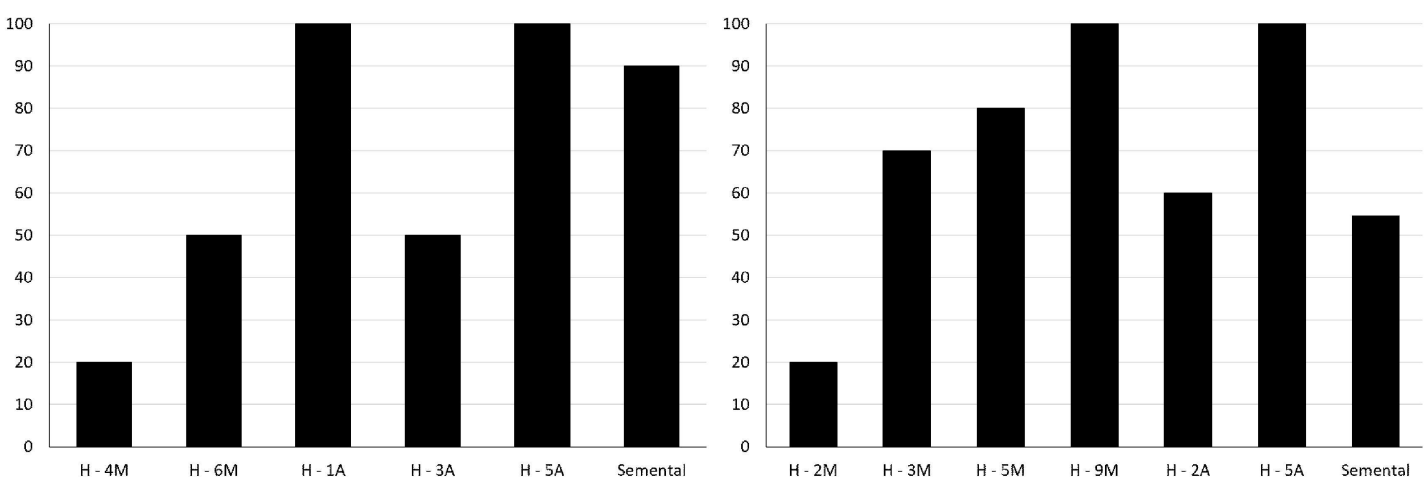

Figura 2. Frecuencia (\%) de cabras positivas a anticuerpos contra Leptospira en dos unidades de producción caprina (Unidad «A», lado izquierdo; Unidad «B», lado derecho), en Apaseo el Alto, municipio de Guanajuato, México, según el grupo etario (2014) (H: hembra; M: meses de edad; A: años de edad) 

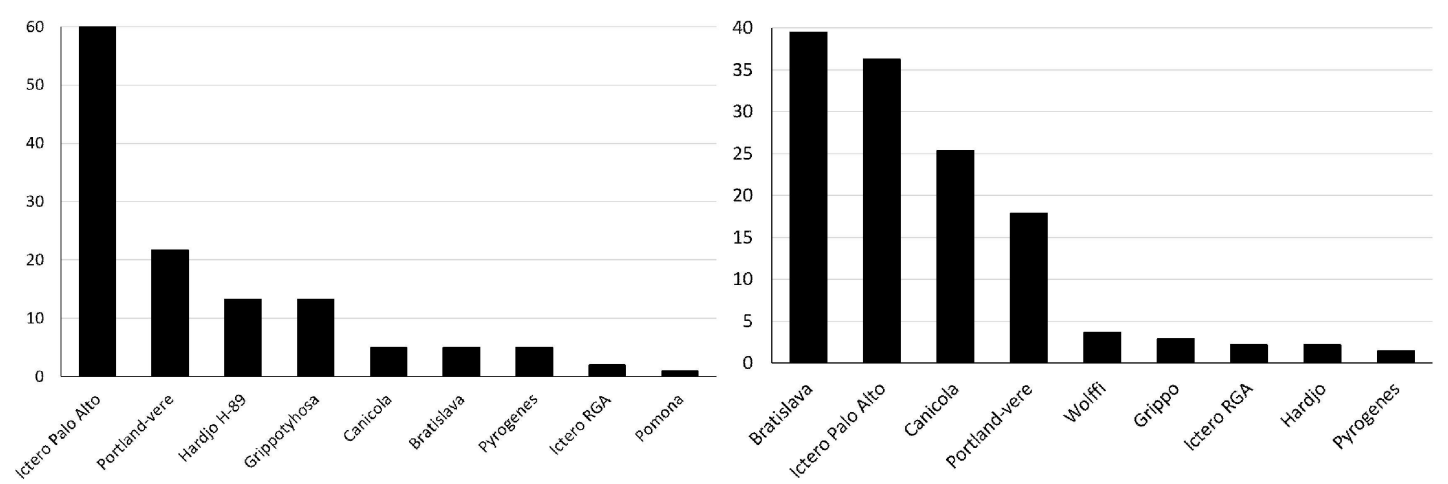

Figura 3. Frecuencia (\%) de cabras positivas a anticuerpos contra leptospirosis, según la serovariedad, en dos unidades de producción caprina (Unidad «A», lado izquierdo; Unidad «B», lado derecho), en Apaseo el Alto, municipio de Guanajuato, México

proceso infeccioso, ya que estos animales nunca fueron vacunados contra Leptospira. Los títulos altos de anticuerpos podrían estar asociados a la presencia del microorganismo y con algunos de los trastornos reproductivos reportados. Se recomienda establecer un programa de vacunación y aplicación de antibióticos para el control de la enfermedad en estas unidades de producción.

\section{Literatura Citada}

1. Acha P, Szyfres B. 2001. Leptospirosis. En: Zoonosis y enfermedades transmisibles comunes al hombre y a los animales. $3^{\circ}$ ed. Washington DC: OPS. p 175-186.

2. Adler B, de la Peña-Moctezuma A. 2010. Leptospira and leptospirosis. Vet Microbiol 140: 287-296. doi: 10.1016/ j.vetmic.2009.03.012

3. Faine S. 1982. Guidelines for the control of leptospirosis. Geneva: Word Health Organization. WHO Offset Publication No. 67. p 171.

4. Gómez M. 2005. Determinación de anticuerpos maternos transferidos a becerros nacidos de vacas vacunadas contra leptospirosis en un rancho de Atilaquia, Hidalgo. Tesis de Licenciatura. México: Univ. Nacional Autónoma de México. 41 p.
5. Hagan W, Bruner D. 1981. Enfermedades infecciosas de los animales domésticos. $4^{\mathrm{a}}$ ed. La Prensa Médica Mexicana. $816 \mathrm{p}$.

6. Lilenbaum W, Morais ZM, Goncales AP, Souza GO, Richtzenhain L, Vasconcellos SA. 2007. First isolation of leptospires from dairy goats in Brazil. Braz J Microbiol 38: 507-510. doi: 10.1590/S1517-83822007000300023

7. Levett P. 2001. Leptospirosis. Clin Microbiol Rev 14: 296-326. doi: 10.1128/ CMR.14.2.296-326.2001

8. Luna AMA, Socci EG, Banda RV, Santos ME, Luna REM, Gavaldón RD. Moles CLP. 2014. Frecuencia de anticuerpos de leptospirosis en ovinos y control de la enfermedad por medio de vacunación. Entorno Ganadero BM Editores No. 66: 101-110.

9. Mallard BA, Dekkers JC, Ireland MJ, Leslie KE, Sharif S, Vankampen CL, Wagter L, Wilkie BN. 1998. Alteration in immune responsiveness during the peripartum period and its ramification on dairy cow and calf health. J Dairy Sci 81: 585-595. doi: 10.3168/jds.S00220302(98)75612-7

10. Manual Merck de Veterinaria. 1988. Leptospirosis. 4a ed. Madrid: Ed Centrum Técnicas. 1918 p. 
11. [NOM] Norma Oficial Mexicana NOM-038-ZOO-1995 Requisitos mínimos para las bacterinas empleadas en la prevención y control de la leptospirosis bovina. 1996. México: Secretaría de Agricultura, Ganadería y Desarrollo Rural. [Internet]. Disponible en: http://dof.gob.mx/nota_detalle. php? $\operatorname{codigo}=4894680 \&$ fecha $=05 / 08 /$ 1996

12. [NOM] Norma Oficial Mexicana NOM-029-SSA2-1999, para la vigilancia epidemiológica, prevención y control de la leptospirosis en el humano. 1999. México: Secretaría de Salud [Internet]. Disponible en: http:// www.salud.gob.mx/unidades/cdi/nom/ 029ssa29.html

13. [NOM] Norma Oficial Mexicana NOM-017-SSA-2012 para la vigilancia epidemiológica. 2012. México: Secretaría de Salud. [Internet]. Disponible en: http://vpn.salud.df.gob.mx:88/transparencia/art 14 frac $1 /$ Normatividad\%20SSPDF/NOM-017-SSA22012.pdf

14. [NOM] Norma Oficial Mexicana PROY-NOM-029-SSA2-2014 para la prevención y control de la leptospirosis en el humano. 2014. México: Secretaría de Salud. [Internet]. Disponible en: http://dof.gob.mx/nota_detalle.- php? codigo $=5422281 \&$ fecha $=07 / 01 /$ 2016\&print $=$ true

15. [OIE] Organización Mundial de Sanidad Animal. 2008. Leptospirosis. París: OIE [Internet]. Disponible en: http:// web.oie.int/eng/normes/MMANUAL/ 2008/pdf/2.01.09_LEPTO.pdf

16. Rebhum W. 1999. Enfermedades del ganado vacuno lecheroZaragoza, España: Acribia. $78 \mathrm{p}$.

17. Seijo A, Romer Y, San Juan J, Prieto $R$, Nogueras M, De Vedia L, Font L, Giamperetti S. 2011. Neumonía aguda de la comunidad y hemorragia pulmonar por leptospirosis en el área metropolitana Buenos Aires. Medicina (B. Aires) 71:127-134.

18. [WHO] World Health Organization. 2013. Zoonoses. Leptospirosis. [Internet]. Disponible en: http://www.who.int/ zoonoses/diseases/leptospirosis/en/

19. Zamora J, Riedemann S. 1986. Consideraciones para la interpretación de la prueba de aglutinación microscópica en el diagnóstico de la leptospirosis bovina. Arch Med Vet 18: 145-147.

20. Zavala J, Cárdenas M, Vado I, Cetina M, Cano J, Laviada H. 2008. Hemorrhagic pulmonary leptospirosis: three cases from the Yucatan peninsula, México. Rev Soc Bras Med Trop 41: 404-408. 